\title{
Interactions of bound multiple solitons in strongly birefringent fibers
}

\author{
Michel W. Chbat \\ Laboratory for Physical Sciences, University of Maryland at College Park, 8050 Greenmead Drive, College Park, Maryland 20740-4000
}

Curtis R. Menyuk

Department of Electrical Engineering, University of Maryland Baltimore County, Baltimore, Maryland 21228-5398

Ivan Glesk and Paul R. Prucnal

Department of Electrical Engineering, Princeton University, Princeton, New Jersey 08544-5263

Received August 8, 1994

\begin{abstract}
We report the observation of the interaction of bound multiple solitons generated by orthogonally polarized, highamplitude pulses in strongly birefringent fibers. For the birefringence used, the threshold amplitude for the interaction is higher than that of the onset of second-order solitons on each axis. The characteristics of the output pulses are in good agreement with the results of a numerical simulation of this interaction. A general investigation of this effect is carried out at high values of birefringence, and it is found numerically that, even though the system is nonintegrable, the description of its evolution appears to be reduceable to a finite number of effective degrees of freedom.
\end{abstract}

Because of their multiple applications, interactions of orthogonally polarized solitons in birefringent fibers have attracted considerable attention. For example, they are used as a basis for efficient all-optical logic gates. $^{1}$ Menyuk has predicted $^{2}$ that for solitons polarized along the principal axes of a moderately birefringent fiber, and above a certain threshold amplitude that depends on the fiber and pulse characteristics, the cross-phase modulation (XPM) can compensate for the walk-off that is due to linear birefringence. Through XPM, the solitons shift their center frequencies, and hence their group velocities, in such a way that they travel as a unit, forming a bound vector soliton. As the birefringence increases, the threshold amplitude will be such that the partial pulses form breathers having two-soliton structures, ${ }^{2}$ and for high values of birefringence the initial partial pulses form larger numbers of bound multiple solitons. We define this regime as strong birefringence. In this Letter we study the characteristics of the interactions of bound multiple solitons generated by high-amplitude pulses in strongly birefringent fibers. We will show that, even though the resulting system is nonintegrable, some qualitative assessment of the evolution of the vector solitons can be made.

If $L_{B}$ is the beat length of the birefringent fiber and $Z_{0}$ is the soliton period, the condition for moderate (or higher) birefringence exists when $Z_{0} / L_{B} \gg 1$. In this case, the normalized equations governing the propagation of two pulses along the principal axes of a birefringent fiber $\operatorname{are}^{3,4}$

$$
\begin{gathered}
i\left(\frac{\partial u}{\partial z}+\delta \frac{\partial u}{\partial t}\right)+\frac{1}{2} \frac{\partial^{2} u}{\partial t^{2}}+\left(|u|^{2}+\frac{2}{3}|v|^{2}\right) u=0, \\
i\left(\frac{\partial v}{\partial z}-\delta \frac{\partial v}{\partial t}\right)+\frac{1}{2} \frac{\partial^{2} v}{\partial t^{2}}+\left(\frac{2}{3}|u|^{2}+|v|^{2}\right) v=0,
\end{gathered}
$$

where $u$ and $v$ are the pulse envelopes along both principal polarizations, $z$ is the distance of propagation, and $t$ is the local time on the pulse. We take the normalization of $t$ to be $t_{c}=\tau /[2 \ln (1+\sqrt{2})]$, where $\tau$ is the FWHM of the pulse intensity. $z$ is normalized by $z_{c}=(2 / \pi) Z_{0}=2 \pi c t_{c}{ }^{2} /\left[\lambda_{0}{ }^{2} D\left(\lambda_{0}\right)\right]$, where $\lambda_{0}$ is the carrier wavelength of the optical pulse, $D\left(\lambda_{0}\right)$ is the fiber dispersion, and $c$ is the speed of light in vacuum. $|u|^{2}$ and $|v|^{2}$ represent the power on the two principal axes and are normalized by $P_{c}=$ $\lambda_{0}{ }^{3} D\left(\lambda_{0}\right) A_{\text {eff }} /\left(4 \pi^{2} c n_{2} t_{c}{ }^{2}\right)$, where $A_{\text {eff }}$ is the effective core area of the fiber and $n_{2}$ is the nonlinear-index coefficient of the fiber. Finally, $\delta$ is the normalized birefringence defined by $\delta=\pi \Delta n t_{c} /\left[\lambda_{0}^{2} D\left(\lambda_{0}\right)\right]$, where $\Delta n$ is the difference between the indices of refraction on both principal axes. All our numerical simulations are based on a numerical integration of Eqs. (1) and (2) using a split-step Fourier-transform technique, with the input condition

$$
u(0, t)=v(0, t)=\frac{a}{\sqrt{2}} \operatorname{sech}(t)
$$

It has been shown numerically ${ }^{2}$ that, for the input condition of Eq. (3) and for values of $\delta<1.0$, the threshold amplitude increases with increasing $\delta$. Various analytical approaches have been considered for this case, and the dependence of the amplitude threshold on $\delta$ was found to be either linear ${ }^{5}$ or nonlinear ${ }^{6}$ and to match to some extent the numerical results. However, these approaches do not give reasonable results for higher values of $\delta$ when the system can be considered as containing more than a single soliton on each polarization axis. Indeed, the critical value of $\delta$ that gives a threshold amplitude corresponding to the onset of second-order solitons is found by numerical simulation to be approximately 


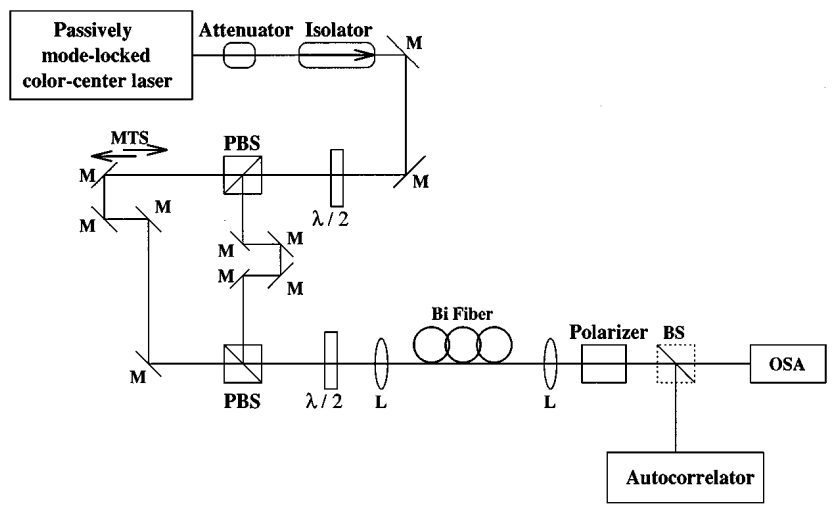

Fig. 1. Experimental setup: M's, mirrors; L's, lenses; MTS, motorized translation stage; BS, beam splitter; PBS's, polarizing beam splitters; $\mathrm{Bi}$, birefringent; $\lambda / 2$ 's, half-wave plates; OSA, optical spectrum analyzer.

$\delta_{c}=1.08$. Furthermore, the experimental observations of the soliton interactions were limited to values of $\delta<1.0 .^{1}$

We carried out an experiment to observe the interactions of high-amplitude solitons for a value of $\delta>\delta_{c}$. Figure 1 shows the experimental setup. A passively mode-locked $\mathrm{NaCl}: \mathrm{OH}^{-}$color-center laser generates nearly transform-limited Gaussian pulses of width $\tau=375 \mathrm{fs}$, with a center wavelength $\lambda_{0}=$ $1.6145 \mu \mathrm{m}$. We used a $16-\mathrm{m}$ length of moderately birefringent fiber having at this wavelength a dispersion $D\left(\lambda_{0}\right)=5.25 \mathrm{ps} \mathrm{km}^{-1} \mathrm{~nm}^{-1}$, an effective core area $A_{\text {eff }}=4.77 \times 10^{-7} \mathrm{~cm}^{2}$, and a linear birefringence $\Delta n=2.40 \times 10^{-5}$, yielding $\delta=1.17$. A combination of a half-wave plate and a pair of polarizing beam splitters was used to produce two orthogonally polarized pulses of adjustable power ratio, which, in this experiment, was maintained at 1 . A second halfwave plate was placed at the fiber input to couple the pulses into the fiber along its principal axes. The polarization extinction ratio was kept between 14:1 and 19:1. The two pulses were temporally coincident at the fiber input, and the timing between them was controlled by a motorized translation stage that permitted a timing resolution of approximately $0.7 \mathrm{fs}$.

We performed a numerical simulation to determine the pulse threshold amplitude for $\delta=1.17$. For these high-amplitude pulses, the spectra have a complex structure that features multiple peaks and a complex evolution as the pulses propagate through the fiber. Therefore the wavelength shift for a given pulse will be accounted for in our simulations by the frequency centroid $\omega_{c},{ }^{2}$ defined as the spectralintensity-weighted average frequency shift from $\omega_{0}=$ $2 \pi c / \lambda_{0}$ on the spectrum. Figures 2(a) and 2(b) show the variation with the distance of propagation of $\omega_{c}(z)$ and the peak time location $t_{p}(z)$, respectively, of the pulse on the fast axis. The values for the slow-axis pulse are opposite these values. The threshold amplitude for the trapping of both solitons is seen to be $a=2.45$, below which $\omega_{c}(z)$ reaches a constant value and $t_{p}(z)$ grows without bounds and above which both $\omega_{c}(z)$ and $t_{p}(z)$ oscillate, indicating that the two orthogonally polarized pulses are trapped. This value of $a$ corresponds to a normalized peak amplitude of
1.73 on each axis. Hence, in the absence of birefringence, a second-order soliton would form. ${ }^{7}$

The attenuator is adjusted in a way such that the total normalized peak power is $a=2.50$. Figure 3 shows the calculated shape, autocorrelation, and spectrum of the fast-axis pulse at the fiber output for $a=2.50$. The pulse is seen to be compressed to a width of $260 \mathrm{fs}$ and the peak wavelength shift is $8.17 \mathrm{~nm}$. Figure 4 shows the measured autocorrelation trace and spectrum of the fast-axis pulse at the fiber output. It is seen that, assuming a hyperbolicsecant shape, the output pulse width is $271 \mathrm{fs}$, and the peak wavelength shift is $8.2 \mathrm{~nm}$, in good agreement with the numerical results.

It is seen from Fig. 2 that, as the bound vector soliton forms, $\omega_{c}(z)$ and $t_{p}(z)$ oscillate rapidly with a spatial frequency that increases with increasing amplitude, with a low-frequency modulation. In the case of low birefringence, typically $\delta<0.6$, the lowfrequency modulation has a negligible effect over a large distance of propagation, and the system behaves as an independent oscillator. Figure 5(a)
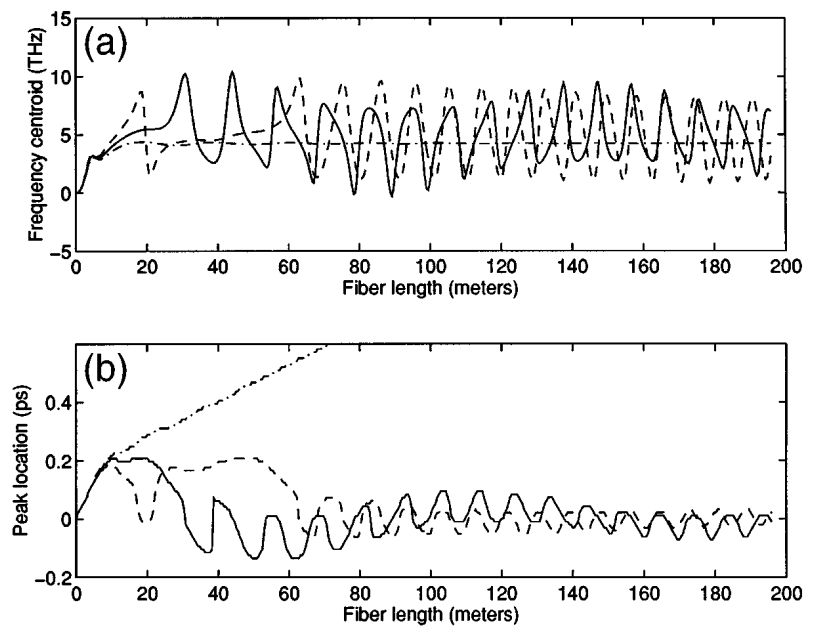

Fig. 2. Numerical results for $\delta=1.17$. Dotted-dashed curves, $a=2.40$; solid curves, $a=2.45$; dashed curves, $a=2.50$. (a) $\omega_{c}(z)$, (b) $t_{p}(z)$.
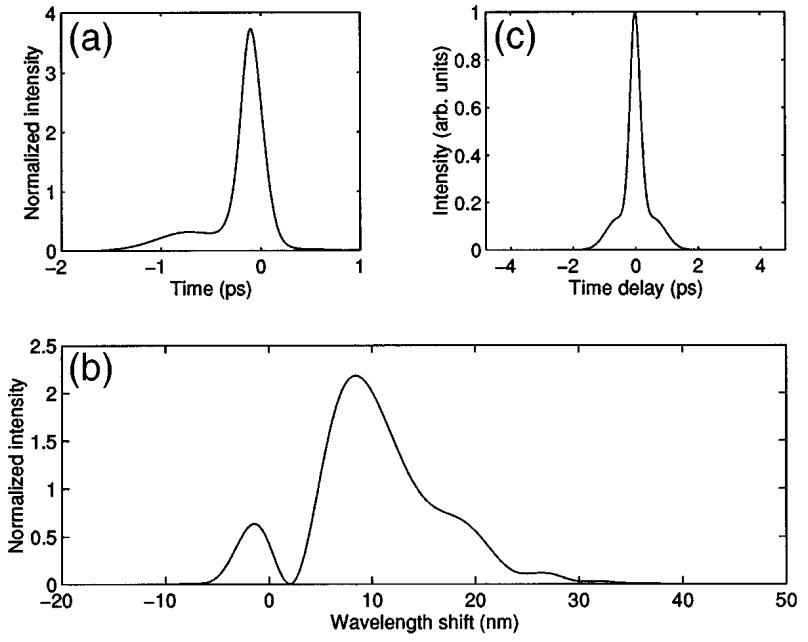

Fig. 3. Numerical results for the fiber output with the experimental parameters: (a) pulse shape, (b) autocorrelation trace, (c) spectrum. 


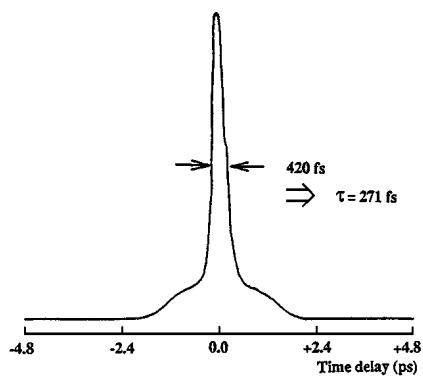

(a)

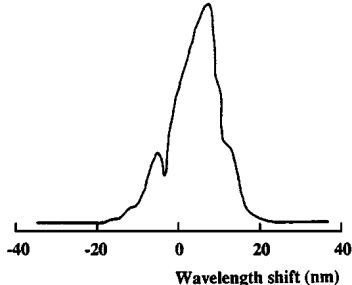

(b)
Fig. 4. Experimental results: (a) output autocorrelation trace, (b) output spectrum. The wavelength shift is measured from the center wavelength of the input pulse $(1614.5 \mathrm{~nm})$.

(a)

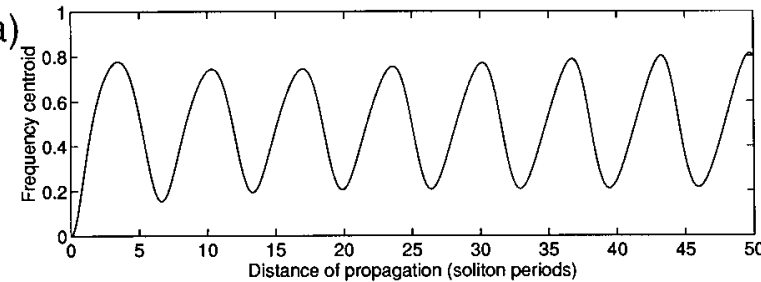

(b)

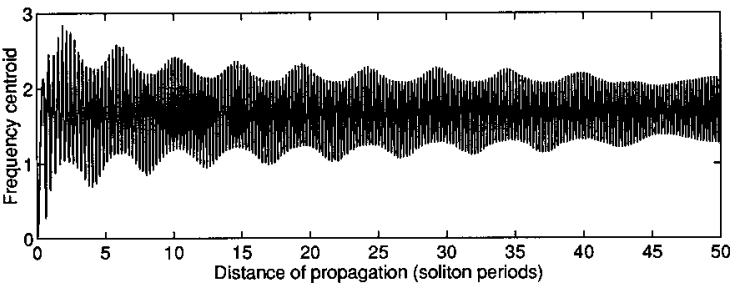

Fig. 5. Numerical results for $\omega_{c}(z)$ : (a) $\delta=0.50$, $a=1.30$; (b) $\delta=2.00, a=4.35$.

shows the variation of $\omega_{c}(z)$ for $\delta=0.50$ and $a=1.30$. Kivshar $^{5}$ gave an analytical account for this motion by describing the soliton as trapped in a harmonic oscillator potential resulting from XPM, with a spatial frequency proportional to the pulse intensity. This approach, which treats single solitons, does not include two major effects: the dispersive waves generated by soliton propagation and the presence of a multiple-soliton structure on each polarization axis. The former effect explains the very slow modulation in Fig. 5(a). Indeed, the nonintegrability of Eqs. (1) and (2) is expected to lead to the continuous generation of a very small amount of dispersive wave radiation beyond all orders during the propagation of an arbitrary input pulse, ${ }^{8}$ which we believe is responsible for the observed rise in the amplitude of the oscillations. In the latter case of bound multiple solitons, each soliton on a principal axis interacts, through XPM, with each of the solitons on the other axis, leading to a complex evolution of the general structure. If $\delta$ is of the order of 1.0 , each partial pulse contains two bound solitons; the system can be described, following Kivshar's approach, as the motion of two coupled harmonic oscillators. This accounts for the presence of two spatial frequencies in Figs. 2. For higher values of birefringence $(\delta \geq 2.0)$, the rapid spatial oscillation of $\omega_{c}(z)$ is modulated by more than two frequencies, as is shown in Fig. 5(b), indicating the presence of a larger number of coupled oscillators. In fact, it can be shown that in this case three or more solitonlike structures are generated on each polarization axis.

This relatively simple motion of the system parameters seems surprising because, as we pointed out, Eqs. (1) and (2) are nonintegrable and therefore can possibly lead to a chaotic evolution of the input vector pulse. However, these equations can be regarded as a Hamiltonian deformation of the nonlinear Schrödinger equation, ${ }^{9}$ which is integrable. Solitons are robust in presence of such deformations, ${ }^{9}$ and, as a result, solitonlike structures can form. Even though the details of the partial pulse structure cannot be quantified, and the system has a priori an infinite number of degrees of freedom that corresponds to the points on the pulse envelope, the results presented above indicate that the system still can be described by a finite number of effective degrees of freedom over a long but finite length. For example, when two bound solitons are present on each principal axis, having amplitudes above threshold, it is seen that two effective degrees of freedom can describe the evolution of the vector soliton. For higher values of birefringence the number of soliton structures increases, and so does the number of the effective degrees of freedom, leading to a complex motion of the soliton structures.

In conclusion, we observed experimentally the interaction of bound multiple solitons generated by two orthogonally polarized high-amplitude pulses in a strongly birefringent fiber. Our results indicate that, in the presence of strong birefringence, initial conditions rapidly evolve into solitonlike structures whose overall motion is governed by a finite set of effective degrees of freedom over a long but finite length.

\section{References}

1. M. N. Islam, Opt. Lett. 15, 417 (1990); M. W. Chbat, B. J. Hong, M. N. Islam, C. E. Soccolich, and P. R. Prucnal, J. Lightwave Technol. 10, 2011 (1992).

2. C. R. Menyuk, J. Opt. Soc. Am. B 5, 392 (1988).

3. C. R. Menyuk, IEEE J. Quantum Electron. QE-23, 174 (1987).

4. In the case of low birefringence, terms oscillating with a normalized spatial frequency of $8 Z_{0} / L_{B}$ are added to Eqs. (1) and (2). Their effect is polarization instability, in which energy is transferred from the fast axis to the slow axis. See, e.g., K. J. Blow, N. J. Doran, and D. Wood, Opt. Lett. 12, 202 (1987).

5. Yu. S. Kivshar, J. Opt. Soc. Am. B 7, 2204 (1990).

6. E. Caglioti, B. Crosignani, and P. Di Porto, Phys. Rev. A 38, 4036 (1988); V. K. Mesentsev and S. K. Turitsyn, Opt. Lett. 17, 1497 (1992); X. D. Cao and C. J. McKinstrie, J. Opt. Soc. Am. B 10, 1202 (1993).

7. J. Satsuma and N. Yajima, Suppl. Prog. Theor. Phys. 55, 284 (1974)

8. H. Segur and M. D. Kruskal, Phys. Rev. Lett. 58, 747 (1987).

9. C. R. Menyuk, J. Opt. Soc. Am. B 10, 1585 (1993). 